\title{
Stabilized Multi-channel ALOHA for Wireless OFDM Networks
}

\author{
Dongxu Shen, Victor O.K. Li \\ Dept. of Elec. and Electronic Engineering \\ Univ. of Hong Kong, Pokfulam Road, Hong Kong \\ \{dxshen, vli\}@eee.hku.hk
}

\begin{abstract}
Multiple access based on orthogonal frequency division multiplexing (OFDM), or OFDMA, enables multiple users to simnitaneoushy access the media by using different subcarriers. This leads to the convenient realization of multi-channel ALOHA, in which each user transmits with a group of subcarriers. In this paper, we first introduce the multi-channel slotted ALOHA algorithm to OFDM, which is called OFDMA-based multichannel ALOHA (OMC-ALOHA). Since ALOHA is an unstable algorithm, we show OMC-ALOHA is also unstable. To solve this stability problem, we extend the pseudo-Bayesian algorithm to achieve stabilized OMC-ALOHA.
\end{abstract}

\section{INTRODUCTION}

In this paper, we consider user random access to a base station. ALOHA is one of the most important algorithms for random access [1]. ALOHA is commonly studied under a single channel assumption, in which user contention happens over only one channel. In the single channel slotted ALOHA, users transmit packets in fixed length time slots. When only one user accesses the time slot, the user packet can be correctly received by the base station. When more than one users transmit at the same time slot, collision occurs. Contending users are informed about the collision, and user packets are retransmitted in future slots.

ALOHA has also been considered for multiple channels (MCALOHA). With multiple channels, more than one packet can be transmitted at the same time, which means higher throughput than the single channel case. In previous work [2]-[6], MCALOHA is achieved through either FDMA [3], [4], [5] (frequency division multiple access) or CDMA [6] (code division multiple access). In FDMA, the frequency spectrum is segmented into channels, while in CDMA, users employ different spreading codes. Both the FDMA and CDMA methods have drawbacks. For FDMA, guard bands have to be inserted between neighboring channels. The use of guard band reduces spectrum utilization. In CDMA, the interference between users is inevitable. Even with orthogonal codes (such as Walsh codes), orthogonality is lost at the base station because of multipath. The interference among users increases packet error rate and reduces the effective throughput. However, there lies another difficulty for MC-ALOHA that is often neglected by previous work. In MC-ALOHA, the selection of a retransmission probability or a transmission channel depends on the collision state of all the channels. The channel state information is carried by the feedback message from the base station. Since a user is linked with one channel at a time, it is hard for the user to be aware of the state of all channels. This difficulty creates much complexity in conventional MC-ALOHA.

The above difficulties are non-existent with OFDM (orthog. onal frequency division multiplexing). The OFDM system is composed of a single carrier divided into multiple subcarriers through fast Fourier transform (FFT). With accurate synchronization, each subcarrier is orthogonal to other subcarriers.
Therefore, multi-channel ALOHA can be achieved through partitioning all subcarriers into subcarrier groups, with each group being a channel for random access. In OFDM based multicharnel ALOHA (OMC-ALOHA), channels are orthogonal, as in FDMA. The difference is that there is no guard band. Most importantly, the feedback message (from the base station) for all the channels can be received by all users. Thus the channel state information is available to all users. This eases the decision for channel selection at the user side.

OFDM is a promising technology for wireless networks. It has been adopted for digital broadcast and in wireless LAN standards. Multiple access based on subcarriers provides an additional option beyond time slots for user random access in OFDM networks. To the best of our knowledge, we are the first to propose multi-channel ALOHA using OFDM.

We consider OFDM used in wireless LAN environment, where users contend to send packets to the base station. In our scheme, all subcarriers are divided into channels. Each channel has the same number of subcarriers. A user selects a channel with equal probability, and waits for the feedback message from the base station. This is similar to ALOHA for a single channel system. However, ALOHA is an unstable algorithm. In single channel ALOHA, one method to achieve stability is to estimate the number of contending users and adjust the retransmission probability. For OMC-ALOHA, it is possible for users to monitor the channel status of all channels, which simplifies retransmission control. We develop a stability algorithm based on the pseudo-Bayesian algorithm in [10].

This paper is organized as follows. In Section II, we review the background of OFDM system. In Section III, we introduce OMC-ALOHA, and analyze its performance. In Section IV, we propose a stability algorithm for OMC-ALOHA. In Section V, we present numerical results. In Section VI, we conclude the paper.

\section{BACKGROUND}

We consider a wireless system using OFDM. The operation of OFDM is sketched in Fig. 1. User data bits are first mapped to complex symbols. The complex symbols are then converted from serial to parallel. The symbol stream, $S_{1}, S_{2}, \ldots, S_{N}$, corresponds to the occupation of subcarriers. Each symbol occupies one subcarrier, as shown in Fig. 1. The IFFT block in Fig. 1 transforms the frequency domain representation into time domain representation. Then the time domain symbols are converted from parallel to serial. A cyclic extension is added to remove the inter symbol interference (ISI) between sequential OFDM symbol blocks. More details of OFDM can be found in [7] and [8].

In OFDMA, the subcarriers are grouped into non-overlapping 


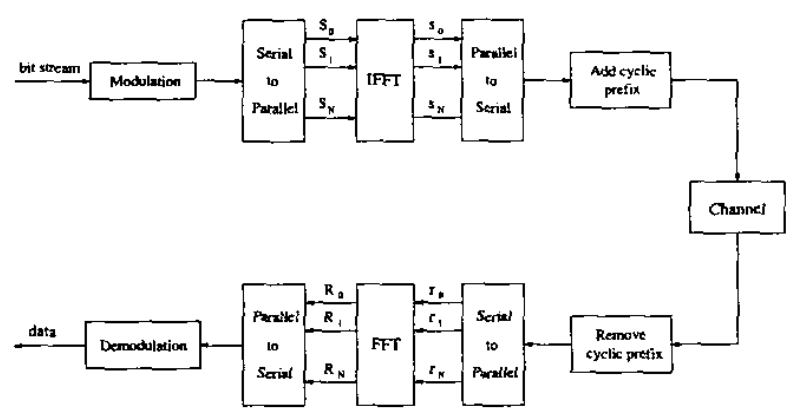

Fig. 1. Operation of OFDM.

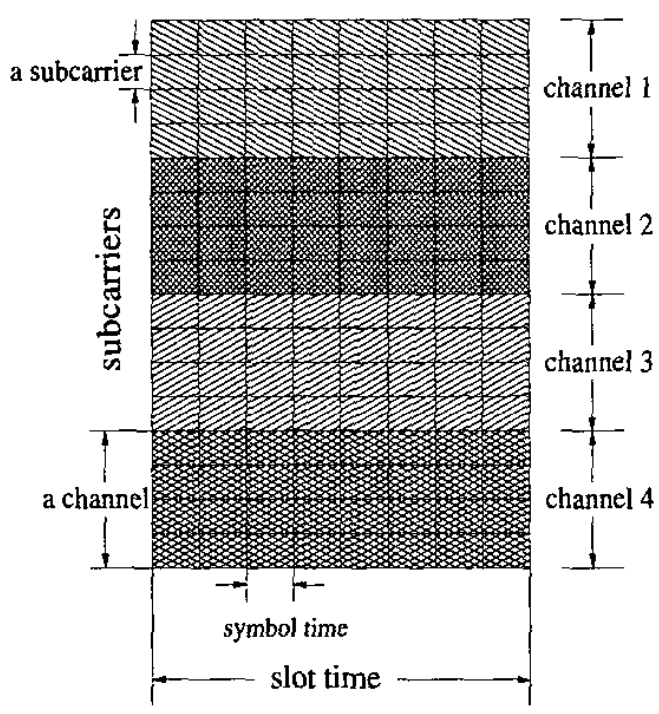

Fig. 2. Channel and slot structure based on OFDM.

sets of subcarriers. We call one set of subcarriers a channel, as shown in Fig. 2. Since subcarriers are orthogonal, different channels are also orthogonal. Further, guard band is not needed, which is an advantage over conventional FDMA.

\section{OFDM-Based MUlti-ChanNEl ALOHA}

\section{A. System Description}

The system we consider is a wireless LAN type environment. Mobile terminals access network resource through a base station. OFDM is used for both uplink (from mobiles to base station) and downlink (from base station to mobiles). Let the total number of carriers be $N$. The $N$ carriers are divided into $M$ channels, each having $\frac{N}{M}$ contiguous subcarriers. Users transmit in fixed length time slots. A time slot lasts for several OFDM symbol time. Figure 2 shows a time slot with 16 subcarriers divided into 4 channels.

At the base station, after receiving uplink user packets, a feedback frame is sent over the downlink. The downlink OFDM symbols are organized into channels with a one-to-one correspondence to each uplink channel. The operations for the uplink

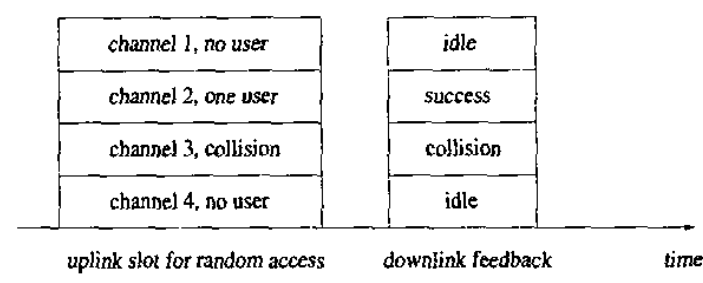

Fig. 3. Illustration of the uplink and downlink.

and downlink are illustrated in Fig. 3. The feedback message for a channel indicates the state of the channel in the last uplink time slot. Specifically, the channel state has three possibilities, idle, success or collision. We do not consider the capture effect.

The downlink feedback frame is received by all users. In OFDM, the data on all subcaniers is available to every user. Thus a user has the information of the feedback messages for all channels. This is different from FDM. In FDM, the channels are physically separated by different frequency bands. Thus a user is unaware of the channel status of other channels that is not operating on the same frequency band. Although it is possible for the feedback messages to be broadcast over all channels, the approach is inefficient, especially when contrasted with OFDM.

In this paper, we follow the common assumptions for ALOHA. Each user has a buffer to store one packet. When more than two users choose the same channel at the same time slot, all packets are lost. The capture effect is not considered. A user with stored packet is called a backlogged user. At a time slot, the retransmission probability for a backlogged packet is $p_{r}$. The number of unbacklogged users having new packet arrival has a Poisson distribution, with arrival rate $\lambda$.

\section{B. OMC-ALOHA Algorithm}

In our scheme, when a user has a packet to transmit, the user has a transmission probability of $p_{r}$. Once a user decides to transmit, the user should choose a channel from all $M$ channels. Let $p_{n}$ be the probability that channel $n$ is chosen. In our OMCALOHA algorithm, we require $p_{n}$ be uniform for all $n$, i.e.,

$$
p_{n}=1 / M, \text { for } 1 \leq n \leq M \text {. }
$$

This design simplifies the channel selection. The central issue is on determining the transmission probability $p_{r}$, which is discussed in the next section.

\section{Performance Analysis}

We first perform the basic performance analysis for OMCALOHA. We assume the number of packet transmissions in one time slot is a Poisson random variable. The average attempt rate is $G$. Note the attempt rate is different from the new packet arrival rate $\lambda$. Since the probability for a user to choose a channel is equal, the number of packet arrivals at a channel is also Poisson with rate $G / M$. Therefore, the system throughput $T_{n}$ at a channel $n$ can be expressed as

$$
T_{\mathrm{n}}=\frac{G}{M} e^{-\frac{G}{M}}
$$


The overall throughput at a time slot is

$$
\begin{aligned}
T & =\sum_{n=1}^{M} T_{n} \\
& =G e^{-\frac{C}{M}} .
\end{aligned}
$$

It is clear from Eq. (1) that the maximal throughput for a channel is achieved when $\frac{G}{M}=1$ (or $\left.G=M\right)$, and $\max \left(T_{n}\right)=e^{-1}$. The maximal throughput of all $M$ channels is $M e^{-1}$, which can be viewed as system capacity limit. Obviously, the maximal new packet rate $\lambda$ should satisfy $\lambda<M e^{-1}$ for a stable system.

\section{STABILIZED MULTl-CHANNEL ALOHA}

\section{A. Basic Idea}

The ALOHA algorithm is an unstable algorithm [9]. At the unstable state, the system throughput is very low, and user packets experience extremely long delay. There has been much research for stabilizing the single channel ALOHA. The key is to determine appropriate transmission probability through the feedback message from the base station. In contrast, little has been done for the stability of multi-channel ALOHA. The stability issue is discussed in [4]. In [5], a backoff algorithm is studied, because the status of other channels is not needed in the backoff algorithm.

For the single channel ALOHA, the feedback message is available to all users since all users share the same media. For MC-ALOHA based on FDMA or CDMA, message for a channel is not directly available to users in other channels. Although backoff algorithm can be implemented for MC-ALOHA [5] even without overall channel information, better performance is expected for algorithms utilizing channel state information. In OFDM based MC-ALOHA, the channels are logical channels, and the feedback messages for all channels are available for all users. Thus the stability control can be greatly simplified.

The stabilization algorithm we propose is based on the pseudo Bayesian algorithm [10]. Before elaborating on our methods, we first briefly introduce the original pseudo Bayesian algorithm, which is designed for the single channel case.

\section{B. Pseudo Bayesian Algorithm}

The pseudo Bayesian algorithm treats new arriving packets as backlogged packets. Let the transmission probability be $p_{r}$. When there are $u$ users having backlogged packets, the overall attempt rate is just

$$
G=u p_{r} .
$$

For the single channel case, we know the maximal throughput is achieved at $G=1$. Therefore, if the number of attempting users is know, $p_{r}$ can be chosen as

$$
p_{r}=\frac{1}{u} .
$$

Since ALOHA is a distributed algorithm, $u$ can only be estimated. The pseudo Bayesian algorithm provides an estimate $\widehat{u}$ for $u$ based on the feedback message from the base station. Let the estimate of $u$ at time slot $k$ be $\widehat{u}_{k}$. At time slot $k+1, \widehat{u}_{k+1}$ is estimated as

$$
\widehat{u}_{k+1}= \begin{cases}\max \left\{\lambda, \widehat{u}_{k}+\lambda-1\right\}, & \text { for } \text { idle or sucess } \\ \widehat{u}_{k}+\lambda+(e-2)^{-1}, & \text { for collision }\end{cases}
$$

Users at slot $k+1$ transmit with probability

$$
p_{r}(k+1)=\min \left\{1,1 / \widehat{u}_{k+1}\right\} .
$$

The new packet arrival rate $\lambda$ is a parameter in (5). Since the maximal throughput for slotted ALOHA is $e^{-1}$, it is required that $\lambda<e^{-1}$. In practice, $\lambda$ is unknown and should be estimated. However, it has been shown [11] that $\lambda$ can be chosen as a fixed value $e^{-1}$, and stability is always achieved.

\section{Pseudo Bayesian Algorithm for OMC-ALOHA}

We extend the pseudo Bayesian algorithm from a single channel to OMC-ALOHA. When there are $M$ channels, obviously the maximal throughput is achieved when the overall attempt rate is $M$. This is because users select a channel with equal probability. When the overall attempt rate is $M$, the attempt rate for each channel is 1 . Therefore, if the total number of attempting users is known as $U$, the transmission probability for a backlogged user can be chosen as

$$
p_{r}=\min \left(1, \frac{M}{U}\right) \text {. }
$$

To estimate $U$, we require each user estimate the number of attempts $\widehat{U}_{n, k+1}$ for a channel $n, n=1, \ldots, M$ at time slot $k+1$. This is because the feedback message for all channels is available for all users, the estimate for every channel is possible. Let $\widehat{U}_{n, k}$ be the estimate at time slot $k$. Then $\widehat{U}_{n, k+1}$ is estimated as

$$
\widehat{U}_{n, k+1}= \begin{cases}\max \left\{\lambda_{a}, \widehat{U}_{n, k}+\lambda_{a}-1\right\}, & \text { for } \text { idle or sucess } \\ \widehat{U}_{n, k}+\lambda_{a}+(e-2)^{-1}, & \text { for collision }\end{cases}
$$

In (8), $\lambda_{a}$ is an estimate of the new packet arrival rate. Then the total attempt rate $\widehat{U}_{k+1}$ is estimated as

$$
\widehat{U}_{k+1}=\sum_{n=1}^{M} \widehat{U}_{n, k+1}
$$

The transmission probability for each user is chosen as

$$
p_{r}(k+1)=\min \left(1, \frac{M}{\widehat{U}_{k+1}}\right) .
$$

\section{NUMERICAL RESULTS}

We consider an OFDM system that is divided into $M$ channels. We assume the number of users is infinite, and the new packet arrival is Poisson with a fixed rate $\lambda^{1}$. This means when the system is unstable, backlog grows to infinity. The rate $\lambda$ is normalized by $M e^{-1}$, which is the system capacity limit. When the arrival rate is higher than $M e^{-1}$, there will be infinite backlogs. Thus system stability is possible only when the normalized arrival rate $\lambda$ is between 0 and 1 . For the simulation of (8), we

${ }^{1}$ Note the arrival rate $\lambda$ is different from the attempt rate $G$. 


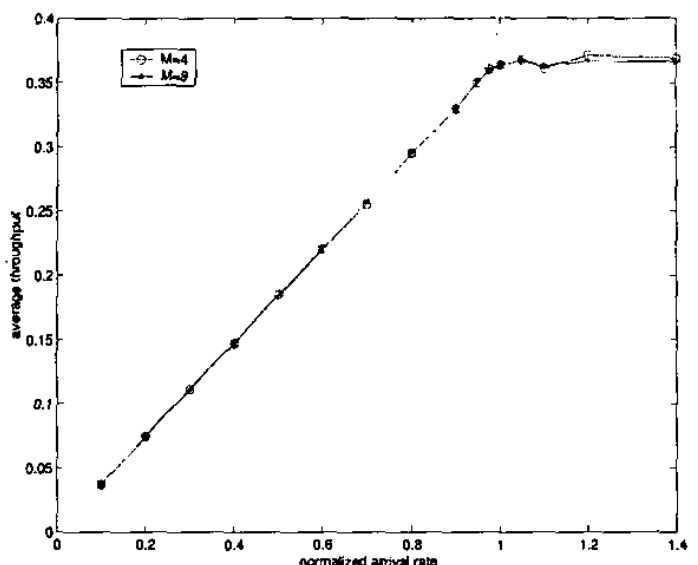

Fig. 4. Average throughput of the stabilized OMC-ALOHA for channels of $M=4$ and $M=8$.

set $\lambda_{a}=e^{-1}$. The performance of the algorithm is characterized by the average throughput and average backlog. The average throughput is the average number of successful channels in a time slot normalized by $M$. The average backlog is calculated as the total number of backlogs divided by the total number of packets. The results are calculated based on the run of 100,000 time slots.

We first show the throughput and backlog of the proposed algorithm. The average throughput for $M=4$ and $M=8$ are plotted in Fig. 4. The average backlog for $M=4$ and $M=8$ are plotted in Fig. 5.

From the two figures, we notice the performance is similar for different number of channels. This is because users select a channel with equal probability, and the arrival rate is normalized so that the average load for each channel is identical. Therefore, each channel will have the same performance.

From Fig. 4, we notice the system throughput is a straight line for $0<\lambda<1$. This is expected. The throughput can be viewed as the departure rate. For a stabilized system, the departure rate equals to the arrival rate. Thus Figure 4 shows the system is stable for all arrival rates of $0<\lambda<1$. In Fig. 4, we also plot the throughput for $\lambda>1$. When $\lambda>1$, the system is unstable with infinite backlogs. However, the throughput can be guaranteed by our stabilization algorithm. From Fig. 4 we notice the throughput is stabilized around the maximal possible rate of $e^{-1}$.

Figure 5 shows the backlog is very small for small $\lambda$. This is a characteristic of ALOHA, which provides low delay when there are few contending users. Figure 5 also demonstrates the delay is not significant for $\lambda$ as high as 0.8 or 0.9 . This is another advantage from our stabilization algorithm which achieves low delay even for high arrival rates. This is superior to the non-stabilized algorithms that are presented later. When $\lambda$ approaches 1 , the delay increase dramatically. This is inevitable because the system operates at almost the capacity limit.

Next we compare the performance of the stabilized OMCALOHA with that of the non-stabilized ALOHA. In the nonstabilized OMC-ALOHA, a new user always transmits immedi-

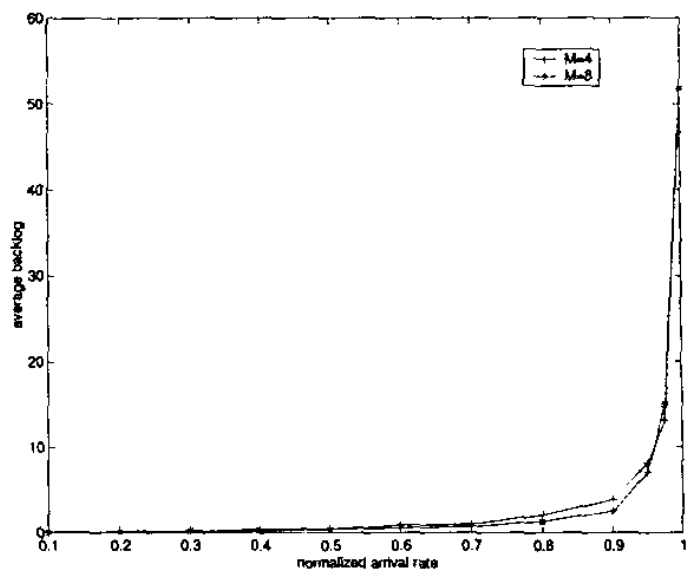

Fig. 5. Average backlog of the stabilized OMC-ALOHA for channels for $M=$ 4 and $M=8$

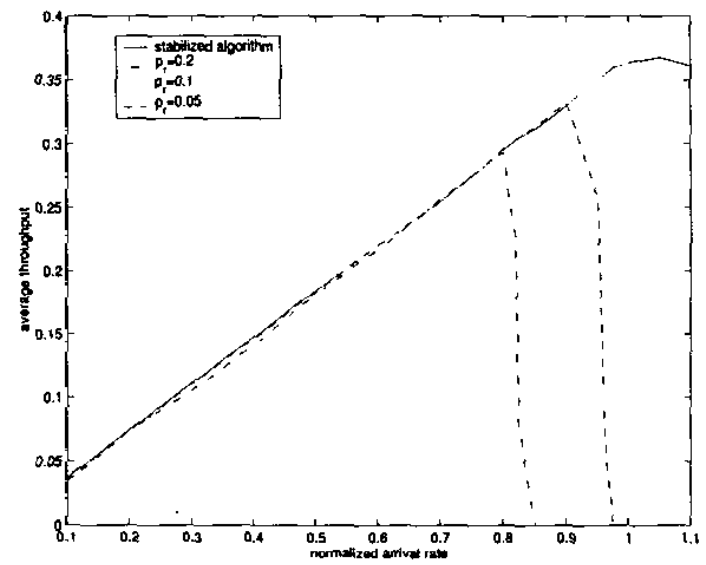

Fig. 6. Comparison of average throughput between stabilized and non-stabilized algorithms.

ately, while a backlogged user transmits with a fixed probability of $p_{r}$. In Fig. 6, we plot the average throughput for $M=4$, $p_{r}=0.2, p_{r}=0.1, p_{r}=0.05$, and the stabilized algorithm. For the non-stabilized algorithm, the average throughput is calculated after 10,000 nuns of time slots.

The non-stabilized algorithms exhibit similar throughput as the stabilized algorithm when the arrival rate is low. However, the throughput of the non-stabilized algorithm degrades dramatically when the arrival rate is higher than a threshold. This threshold depends on the retransmission probability $p_{r}$. From our experiment, the system is very likely to be unstable when $\lambda>0.8$ for $p_{r}=0.2, \lambda>0.85$ for $p_{r}=0.1$, and $\lambda>0.9$ for $p_{r}=0.05$. Thus, a lower $p_{r}$ leads to a more stable system. However, even with a small $\lambda$, stability is not guaranteed by the non-stabilized algorithms.

For the non-stabilized OMC-ALOHA, the system can easily enter the non-stable state for high arrival rates. Since we assume there is no limit on new arrivals, the number of backlog can be- 


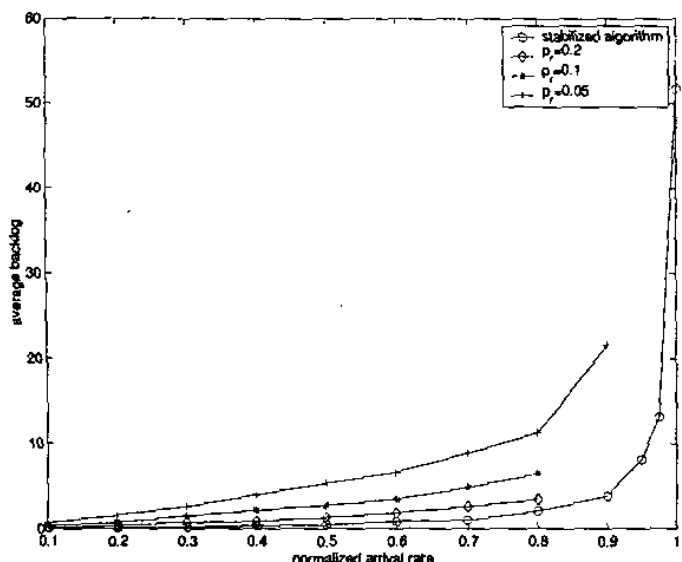

Fig. 7. Comparison of average backlog between stabilized and non-stabilized algorithms.

come infinite when the system departure rate is lower than the arrival rate. Because the attempt rate is a fixed portion of backlog plus the new arrival rate, the attempt rate also becomes infinite with the backlog. Thus collision occurs for every channel at every time slot, and system throughput eventually tends to 0 .

In Fig. 7, we show the average backlog for the above four cases. It is clear that the stabilized algorithm is the best. When $p_{r}$ is chosen to be very small, the non-stabilized algorithm can maintain stability at high arrival rate. For example, when $p_{r}=0.05$, the maximal $\lambda$ can be 0.9 when system is stable. However, the average backlog is much higher than other values of $p_{r}$. Therefore, the stability and backlog optimization are two conflicting factors for the non-stabilized algorithm. However, for the stabilized OMC-ALOHA, the two factors can be jointly optimized, i.e., the algorithm is able to maintain stability with low backlog.

From our experiments we conclude that the stabilized OMCALOHA can achieved much better throughput and backlog performance than the non-stabilized algorithm. The system throughput can be maintained even when the arrival rate exceeds system capacity.

\section{CONCLUSION}

In this paper, we propose a multi-channel ALOHA algorithm based on OFDM. In OMC-ALOHA, multiple channels are formed through partitioning subcarriers of an OFDM symbol. A user selects a channel with equal probability. To stabilize the algorithm, we extend the pseudo Bayesian algorithm from a single channel to OMC-ALOHA. The transmission probability for a user is calculated through estimating the number of all contending users. From numerical experiments, we show that the performance of the stabilized algorithm is much better than the non-stabilized algorithm. With the stabilized algorithm, the system is always stable when the arrival rate is less system capacity. Even when arrival rate is higher than capacity, system throughput can still be guaranteed.

\section{REFERENCES}

[1] N. Abramson, "The ALOHA system-another alternative for computer communications," Proc. Fall Joint Comput. Conf., AFIPS Conf., pp. 37.

[2] W. Yue, "The effect of capture on performance of multichannel slotted ALOHA systems," IEEE Trans. Commun., vol. 39, pp. 818-822, June 1991.

[3] Z. Zhang, Y.-3. Liu, "Multichannel ALOHA data networks for personal communications services (PCS)," IEEE Proc. Globecom "92, 1992, vol. 1, pp. 21-25.

[4] I. E. Pountourakis, E. D. Sykas, "Analysis, stability and optimization of ALOHA-type protocols for multi-channel networks," Comput. Commun., vol. 15 , no. 10, pp. 621-29, December 1992.

[5] M. S. Alam, A. E. Hossain, "Throughput analysis of a multichannel slotted-ALOHA protocol in short-haul communication environment for an exponential backoff retransmission scheme," Proc. International Conf. on Information, Commun. and Signal Processing (ICICS) '97, Singapore, 1997, pp. 1034-38.

[6] F. L. Lo, T S. Ng, T. I. Yuk, "Delay throughput comparison of single and multi-channel slotted ALOHA networks," Proc. International Conf. on Commun. Tech. (CCCT) '96, Beijing, PRC, 1996, pp. 934-37.

[7] T. Keller, L. Hanzo, "Adaptive nulticarrier modulation: A convenient framework for time-frequency processing in wireless communications," Proc, IEEE, vol. 88, pp. 61 I-40, May 2000.

[8] R. vas Nee, R. Prasad, OFDM for Wireless Multimedia Communications, Artech House, 2000.

[9] D. Bertsekas, R. Gallager, Dato Networks, Prentice Hall, 1992.

[10] R. L. Rivest, Network Control by Bayesian Broadcast (Report MIT/LCS/TM-285), Cambridge, MA: MIT, Lab. for Computer Science.

[11] J. N. Tsitsiklis, "Analysis of a multiaccess control scheme," IEEE Trans. Automat. Control, AC-12, pp. 1017-20, 1987. 